\title{
EVALUATION OF VERSIONS OF ELECTRIC POWER GRID REPAIR SCHEMES FROM THE RESULTS OF STRUCTURAL RELIABILITY CALCULATIONS
}

\author{
P. V. Toshchakov, ${ }^{1}$ O. M. Kotov, ${ }^{2}$ and A. F. Kostarev ${ }^{2}$
}

Translated from Élektricheskie Stantsii, No. 7, July 2012, pp. 56 - 62.

\begin{abstract}
A procedure for processing repair claims using the results of an estimate of the structural reliability of the electric grid, the results of the approval of algorithms using the example of a test model, and the principles for producing short-term charts of the repairs of electric-grid equipment, is presented.
\end{abstract}

Keywords: electric grid, repair operating period, structural reliability, reliability indicators, breakdown, operative switchings, switching state tree.

Estimating the reliability of ways of repairing the electric grid is one of the most complex problems of short-term planning of the operation of an electric power system. When solving it, it is necessary to coordinate repair operations of the generating and network structures of the power system, and also to match the circuit-operating conditions for carrying out repairs on the electric grid equipment.

The present paper presents a brief description of a method of processing repair claims, based on the results of an estimate of the structural reliability, and the main results of testing it on an actual $500 \mathrm{kV}$ electric grid.

The calculations were carried out for versions both of single repairs of the electrical equipment, and for combinations of them. From the results of the investigations we developed recommendations on drawing up short-term charts of the repairs of electrical network equipment, capable of increasing the circuit reliability of an electric-power system.

Basic statements of the procedure. The procedure for processing dispatcher claims that repairs of the electric grid equipment have been completed uses an estimate of the structural reliability of the switching states of the electric grid circuit, carried out using the "Struna" software. The software is designed to calculate the quantitative characteristics of the structural reliability of complex electric systems. It is possible to estimate the loss of any combinations of examined units and to take into account deliberate disconnections.

The methods used in the "Struna" software ignore operating limitations, but enable one to estimate fairly accurately the structural properties of the electric grid being considered

\footnotetext{
1 JSC "System Operator of the United Power System,", ODU Ural, Yekaterinburg, Russia.

2 B. N. Yel'tsin Ural Federal University, Yekaterinburg, Russia.
}

[1]. The initial information is the network circuit, the hazardous nature of the components which make up the circuit, and node-sources. It is assumed here that any node of the circuit (henceforth the observed node) is in an operating state when there is at least one operating path to the node-source. Breakdowns of the circuit components are divided into two types, depending on their effect on the nodes - breakdowns, which lead to the need to carry out emergency-restoration repairs ("cutoff" type breakdowns - CTB), and breakdowns which are eliminated using operative switchings ("short-circuit" type breakdowns — STB). The short-circuit type breakdowns include breakdowns due to non-disconnection by the circuit breakers of short circuits that are external with respect to the node considered, and also breakdowns of the circuit breakers in the supply subsystems (as a rule, busbar-connecting or sectioned subsystems) [2, 3].

In addition to the reliability indicators for the observed nodes of the electric grid, quantities which estimate the simultaneous failures of two or more nodes can be calculated. In the calculations described, losses of the $500 / 220 \mathrm{kV}$ connections at substations of the test circuit were so estimated. To do this, an additional node and branches, connected to the nodes, are added to the circuit for each such substation, which model the autotransformer or autotransformer group. These branches, being absolutely reliable, in addition ensure the transit of power only in the direction of the additional node. Hence, the node considered only changes into a breakdown state when all the nodes adjacent to it are also in a breakdown state. Total loss of the connection of the corresponding $500 / 220 \mathrm{kV}$ substation corresponds to the breakdown state of this node.

As a result of a calculation using the "Struna" software, a fairly large set of quantitative indicators is obtained, charac- 


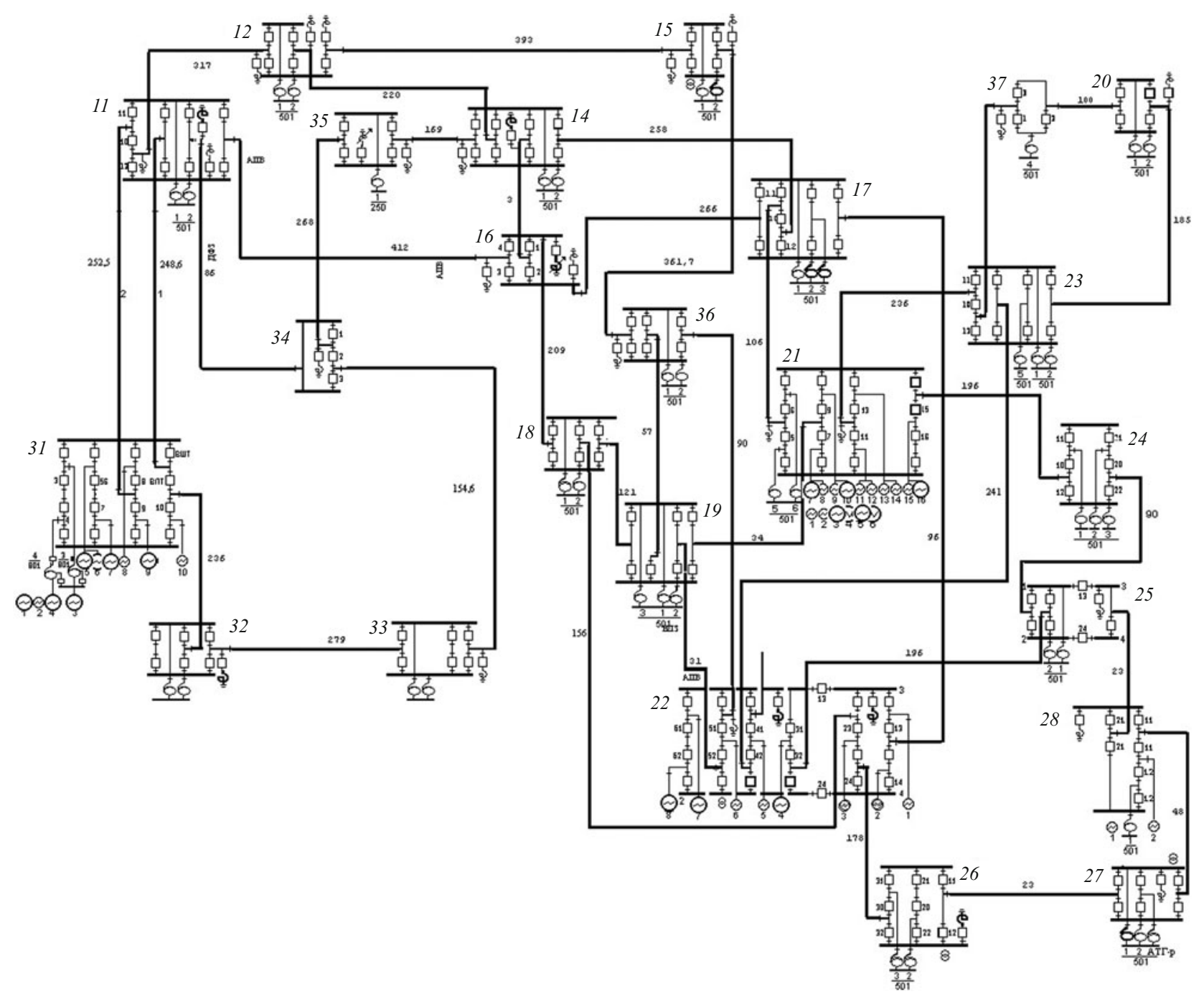

Fig. 1. Theoretical model of the $500 \mathrm{kV}$ grid.

terizing the structural reliability of the nodes of the electric circuit, the main ones of which are the probability and intensity of breakdowns. To reduce this volume, the set of observed nodes is limited by supplementary nodes (the number of which is equal to the number of substations), and a set of total indicators - the failure frequencies (both individually with the CTB and SCTB models and the total numbers).

Finally, the integrated parameter, conditionally called the estimated reliability index (ERI), is set up corresponding to the version of the calculated circuit. The ERI is calculated by adding the frequencies of breakdowns of the observed nodes with weights, equal to the powers of the substation load in one of the characteristic modes of operation (for example, the maximum winter load), and is measured in megawatts per year:

$$
\mathrm{ERI}=\sum f_{x} P_{x},
$$

where $x$ is the number of the substation, $f_{x}$ is the node breakdown frequency, which models simultaneous breakdown of the busbars or sections of the substation, and $P_{x}$ is the total load of the autotransformers or autotransformer groups of the substation $x$ according to monitoring measurement data.

The test scheme. As is well known, electric power grids are complex-closed hierarchical systems, which require adequate methods of analysis, including in problems of choosing the topology and composition of the electric grid equipment. In the course of the repair operating period, the need arises to solve a multifactor problem of coordinating and matching the equipment repair schedules, the modes of operation of which are interconnected. When choosing sets of repairs one must avoid any dangerous limitations on the consumption or load modes of operation of the generating equipment.

The procedure for estimating versions of repair schemes from the results of calculations of the structural reliability is approved on the basis of initial data, representing a model of a fragment of the existing $500 \mathrm{kV}$ electric grid, including 36 overhead loads and 24 distributors. The test scheme is shown in Fig. 1. The overall size of the theoretical circuit is 398 branches and 160 nodes. 


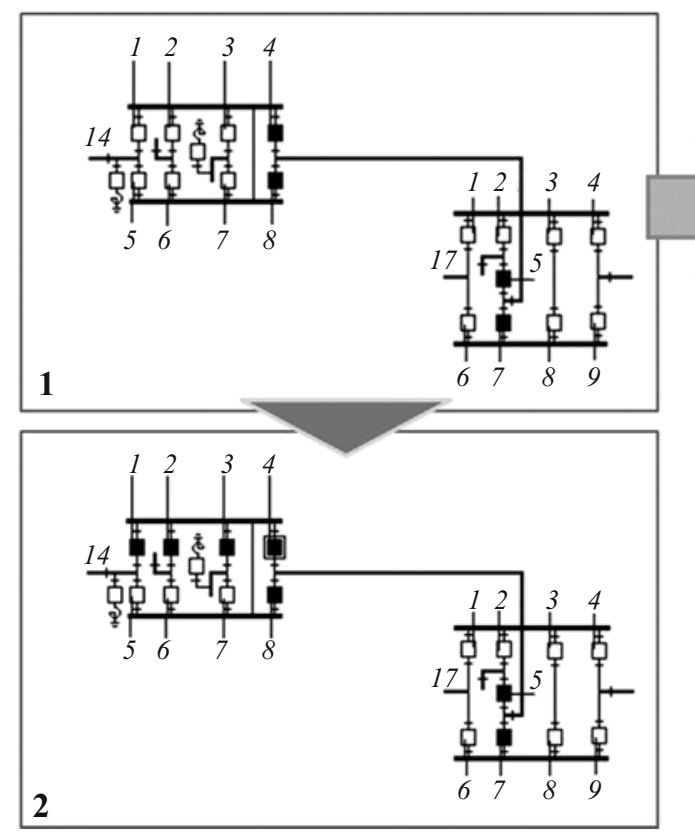

The initial basic data for performing the calculations are the reliability parameters of each component of the electric grid, obtained using statistical data on equipment breakdowns [4]. The set of initial parameters for all the components considered acts as a database, used for calculations of the reliability indicators for the electric grid network. The means for storing statistical data for further action and for extending the database of initial reliability parameters of the grid equipment can also provide monitoring and diagnostics of the operation of the electrical equipment, employed in different grid operating periods, and also the acquisition of information on cases of breakdown of normal operation of the electric grid components in different emergency situations.

The construction of versions of repair schemes. The state of the electric grid directly during operative switching when the $500 \mathrm{kV}$ electric transmission line is being repaired, is modeled by eliminating from the model the normal scheme of disconnected components - power circuit breakers from both sides. The total number of circuit breakers may be different, depending on the substation circuit, which connects the line being repaired. There is therefore a risk that one of the switched circuit breakers will fail, which, in turn, may lead to the need to localize the damaged component with a "deeper" change in the electric grid circuit. This fact is the basis for setting up a set of design versions.

In order to carry out operative switching successfully, the network is again returned to the initial state, with the exception of the disconnected transmission line and the corresponding switching apparatus. The change in the initial normal circuit during the process of repairing several power transmission lines is carried out in succession, with the condition that often switching for repairing the next line only

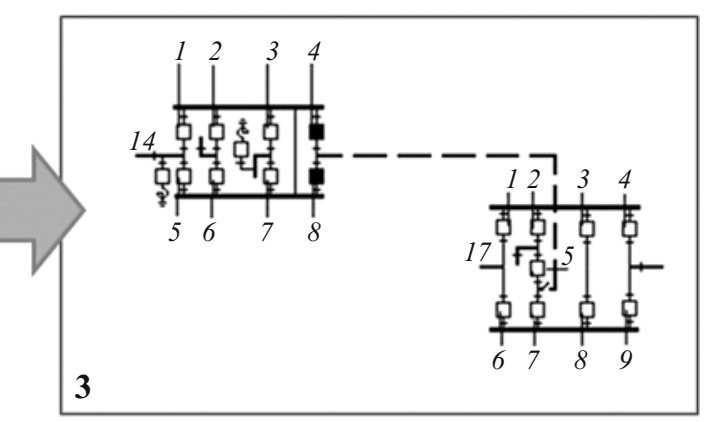

Fig. 2. Example of versions of the switching states when repairing overhead line $17-14$.

begins when the repair of the previous line has been successfully completed.

Consequently, when processing repair statements, three groups of versions of the theoretical circuits are drawn up:

- the first group consists of circuits obtained assuming successful disconnection of the corresponding line from both ends with the corresponding power circuit breakers;

- the second group consists of circuits which model breakdowns when one of the adjacent circuit breaker lines has been disconnected, leading to the need for its localization and repair;

- the second group consists of versions which model a fragment of the electric grid circuit with a disconnected power transmission line over an extended period of repair activity.

The set of three versions of the switching states for repairing a specific line gives a fully adequate picture of the change in the electric network during the repair operating period. In Fig. 2 we show an example of the formation of the versions of the switching states when considering a claim for a repair of line $17-14$. Version 1 corresponds to successful disconnection of the line considered, version 2 corresponds to failure of circuit breaker No. 4 of substation 14, and version 3 corresponds to disconnection of line $17-14$ for carrying out a prolonged repair.

Approval of the technique. At the first stage, in order to rank the versions of the electric grid circuits, we calculated the reliability indicators for circuit situations which simulate successful repair of each electric transmission line successively of those in the test model.

In Fig. 3 we show a histogram of the estimated reliability indicators of the electric grid scheme. For each version, which models the repair of the corresponding line, there is a column on the histogram, the height of which corresponds to 


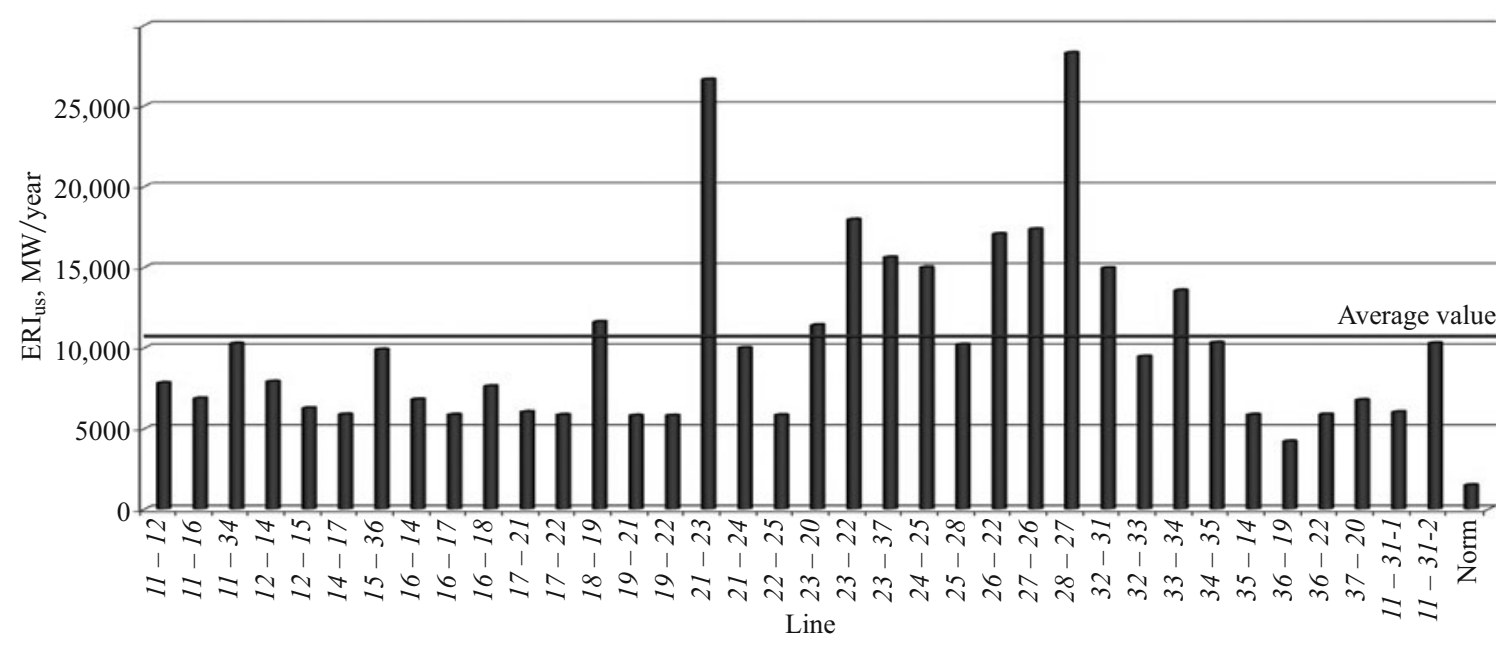

Fig. 3. Histogram of the estimated reliability indicators of a circuit of the electric grid: the overhead line is denoted by the numbers of the substations at the beginning and end of the line.

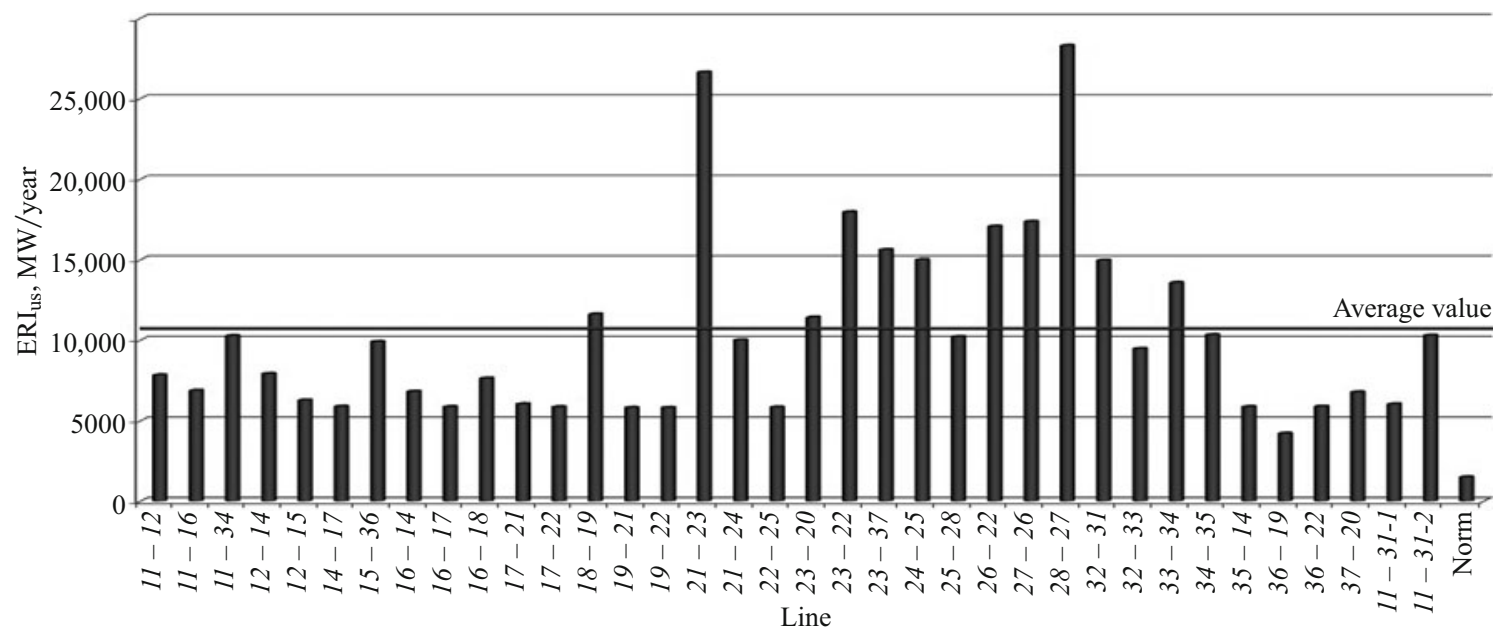

Fig. 4. Histogram of the estimated reliability indicators of the circuit of the grid for single repairs of the overhead line when one of the circuit breakers fails.

the estimated reliability indicator. The lines of the test circuit, denoted by the substation numbers, serve to identify the repaired version of the network considered.

The results of this stage enable us to conclude that, even in the case of a successful repair of the transmission line, the electric grid is weakened from the point of view of circuit reliability to a different degree. A significant part of the disconnections considered have no appreciable effect on the weakening of the structure of the grid. To select the transmission lines, the repair of which considerably reduces the reliability of the network being analyzed, we introduced a system of relative indicators, and we chose the values which exceed the average of all the results obtained. Finally, we formed a group of lines, disconnection of which reduces the reliability of the grid as a whole to the greatest extent. The repair of these lines must be specially monitored by the operational and dispatcher personnel. At the next stage of the analysis we estimated the unfavorable development of events when operative disconnections were carried out. If, when attempting to repair a line, one of the switching circuit breakers fails, it is necessary to disconnect its standby circuit breakers. It is necessary to take into account the repaired circuits "loaded" in this manner since the risk of the occurrence of a similar situation is present for any interference in the normal operation of the switchgear. In Fig. 4 we show a histogram for the estimated reliability indicators of the electric grid circuit assuming an unsuccessful repair in the lines $\left(E R I_{\text {us }}\right)$. ERI $I_{\text {us }}$ is calculated by summing the ERI over all versions of the failures of switchgear adjacent to the disconnected line (measured in megawatts per year):

$$
\mathrm{ERI}_{u s}=\sum \mathrm{ERI}_{m}
$$

where $\mathrm{ERI}_{m}$ is the estimated reliability indicator of the circuit version when circuit breaker $m$ fails. 
TABLE 1

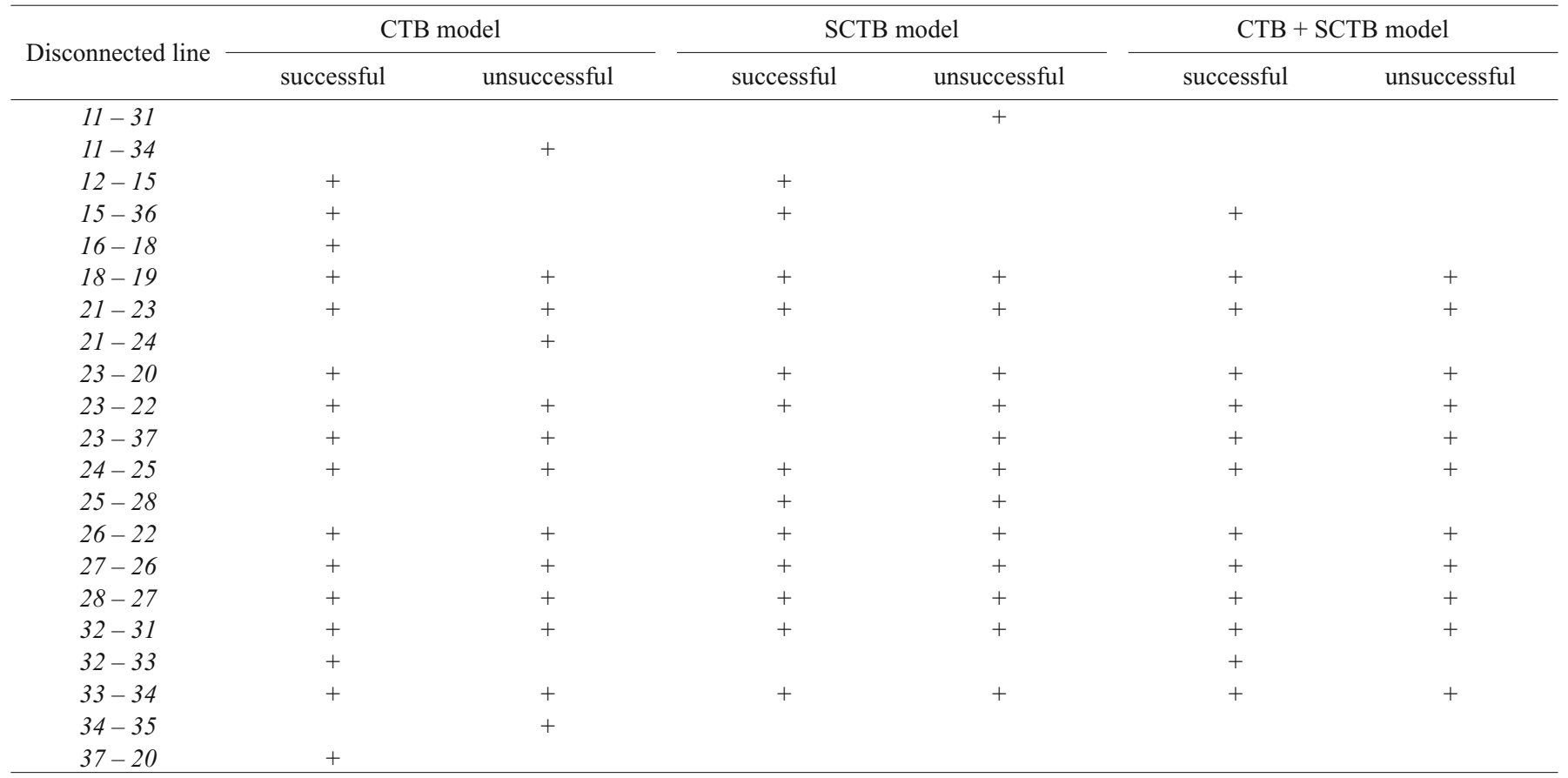

In order to obtain a set of lines, disconnection of which weakens the circuit to the greatest extent, it is necessary to analyze the correlation of the results obtained using different models of the breakdown for the successful and unsuccessful withdrawal of the line for repair. Table 1 shows the entire list of lines which, when disconnected, the reliability indices of the circuit of the grid in one case or another turned out to be higher than the average value. When this condition is satisfied, the line is given a "+" sign in the corresponding column of Table 1.

An analysis of the results confirms the assumption that the reliability indicators, when a line is successfully removed for repair, and the overall indicators for situations when there is an unsuccessful development of events, correlate with one another. As follows from the data in Table 1, the estimated reliability indicator for repairs of removed lines stably exceeds the average value for each method of calculation and is practically identical with the results obtained by adding the frequencies of "cutoff" type breakdowns and "short circuit" type breakdowns (CTB and SCTB) when a line is unsuccessfully removed for repair (the last column).

Not all the versions of the repair schemes in the final selection, noted as having an indicator higher than the average for each method of calculation, also turned out to be most significant for the reliability of the electric grid circuit. For a further analysis we chose as the main ones the results of a calculation of the estimated reliability indicators for the CTB + SCTB model, assuming unsuccessful disconnection of the lines for repair.
From the whole list of lines, we selected 11 transmission lines, requiring increased attention when planning their disconnection when compiling short-term and long-term repair charts. The removal of any of these lines for repair considerably weakens the structure of the electric grid and increases the risk of having to limit power supplies to consumers.

One of the subproblems in short-term planning is estimating the degree of reliability of the operation of the electric grid when different repairs are combined.

During the course of a further investigation, we analyzed the electric grid for simulating double repairs. We noted from the results of preliminary calculations the effect on the grid reliability of removing the first line for repairs in the cases of successful and unsuccessful removal of the second line. It was established that, for equal requirements regarding operating limitations, a number of combinations of double repairs reduce the reliability of the grid to a different extent. We estimated the reliability indicators for double repairs for two ranges of the maximum permissible power return flow. The ranges were chosen deliberately for the greatest and least established values, in order to verify the adequacy of the procedure in different operating situations.

A decisive factor in this case is the presence in the combination of one of the previously removed lines, according to the results of a calculation for single repairs. The location in the circuit of the lines under repair also has an effect on the value of the reliability indicators obtained. For a minor removal of the lines considered, the reliability is reduced to a greater extent than when lines situated in different parts of the circuit are under repair. 
When modeling the repair of two or more lines, we successively analyzed several stages of the structural changes of the test circuit of the electric grid, including successful and unsuccessful disconnection of the electric power lines.

As a result we obtained considerable differences in the estimated indicators for successful and unsuccessful repairs, which indicates the effect of a succession of line disconnections. In the framework of short-term planning, by changing the order in which lines are removed for repair, one can reduce the maxima of the calculated reliability indicators and, consequently, also reduce the risk of technological breakdowns occurring.

Switching state tree. The calculated estimated indicators for all stages of operative switchings during the course of the repair period, including for alternative versions, were grouped in the form of a switching state tree. An example of the switching state tree is shown in Fig. 5.

The switching state tree is a structure, consisting of the calculated situations, due to the removal for repair of the main grid equipment in the order in which operative switchings are carried out. Successive stages of the state tree are subdivided, as previously described in the procedure, into periods of normal operation, operative switchings and protracted repairs. An alternative choice of the transmission line for repair defines the vertical gradation. For the period of operative switchings, two estimated reliability indicators are given for successful and unsuccessful removal for repair. The "repair period" (RP) state models the assembled state of the substation circuit during prolonged repairs, which can take place, as a rule, when there is a line disconnecting switch.

The main rule in setting up the state tree is that the number of lines removed in a single analyzed short-term period remains constant, and, as a consequence, the final state of normal operation is always characterized by the same estimated reliability indicator for all outcomes. In the course of time, the strategy of carrying out repairs from stage to stage can be changed, including as a result of the occurrence of operational limitations. In such cases the change in the state switching tree enables one to estimate adequately the level of reliability of the grid at the current instant of time and during several stages of operative switchings ahead to estimate possible directions in which events may develop. Finally, the results obtained enable one to optimize the order in which line repairs are carried out.

It is clear from the results of the above investigations that the primary line repair that reduces the circuit reliability to the greatest extent will be the structure of the grid during the period of operative switchings and the repair period, that is preferable from the point of view of maintaining the

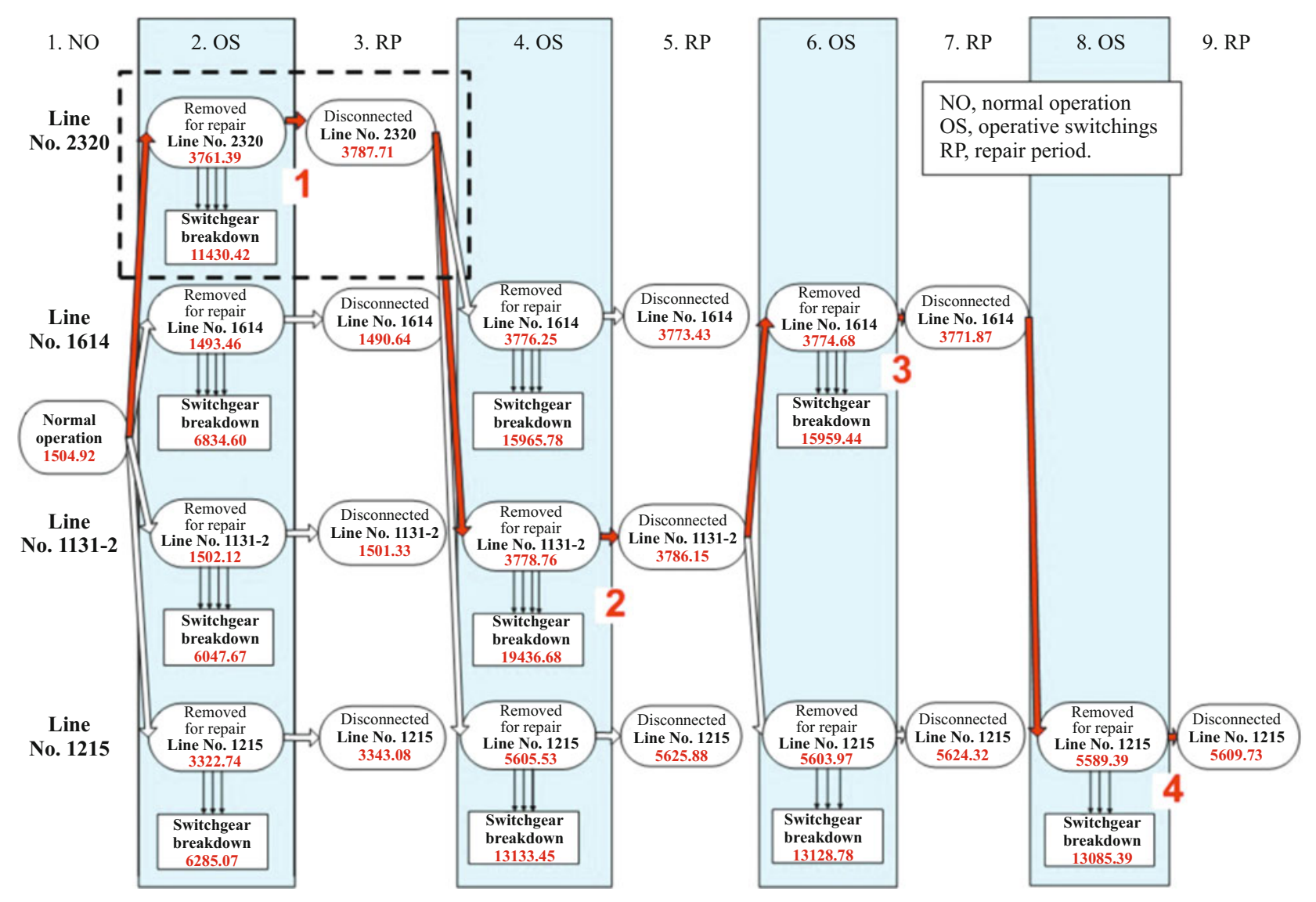

Fig. 5. Switching state tree. 
required level of reliability. If the removal of such a line for repair is unsuccessful, the possibility remains of postponing the repair of the remaining lines to a later date. On the other hand, the repair of such a line can be postponed to another period, in which the circuit-operating situation is less "burdensome." The results of calculations of the estimated reliability indicators may also serve as a basis for a different combination of repairs.

\section{CONCLUSIONS}

1. A consideration of the results of a structural analysis in addition to operating limitations helps to increase the level of reliability of the electric grid when different kinds of repairs are being carried out.

2. The results of a check of the proposed procedure has shown that it is well founded and can be used as a means of analyzing various circuit situations.

3. Further improvement and adaptation of the procedure, in accordance with modern requirements for increasing the operating reliability of electric power systems, is desirable.
4. To reduce the time involved in the calculations and in processing the results, it is necessary to develop specialized software as part of a universal system for considering dispatcher statements regarding the change in the technological state of the equipment.

\section{REFERENCES}

1. D. A. Arzamastsev and V. P. Oboskalov, Calculation of the Structural Reliability Indicators of Power Systems. A Textbook [in Russian], Izd. UPI, Sverdlovsk (1986).

2. V. P. Oboskalov, O. M. Kotov and A. S. Dulesov, "The structural reliability of electric power supply systems with 'short-circuit' breakdowns," Izv. Vuzov. Énergetika, No. 2 (1986).

3. V. P. Oboskalov, I. L. Kirpikova and O. M. Kotov, "The use of the method of basis sections to estimate the functional and structural reliability of electric power systems," Izv. AN SSSR. Énerget. Transport, No. 2 (1989).

4. Yu. N. Rudenko (ed.), The Reliability of Power Systems and Their Equipment. Vol. 2 [in Russian], Énergoatomizdat, Moscow (2000). 